\title{
Site-directed immobilization antibody for alpha- fetoprotein detection by optical biosensor
}

\section{Chenghong Huang, QingLin Yang, Fayi Song, Nan Chen, Xiaoling Liao, Bo Yao, ShiTong Zhang, Yanyan Chen \& Gang Jin}

To cite this article: Chenghong Huang, QingLin Yang, Fayi Song, Nan Chen, Xiaoling Liao, Bo Yao, ShiTong Zhang, Yanyan Chen \& Gang Jin (2016) Site-directed immobilization antibody for alpha-fetoprotein detection by optical biosensor, Integrated Ferroelectrics, 171:1, 70-78, DOI: 10.1080/10584587.2016.1172004

To link to this article: http://dx.doi.org/10.1080/10584587.2016.1172004

曲 Published online: 27 May 2016.

Submit your article to this journal $\pi$

Џ Article views: 10

View related articles $\smile$

View Crossmark data $\nearrow$ 


\title{
Site-directed immobilization antibody for alpha-fetoprotein detection by optical biosensor
}

\author{
Chenghong Huang ${ }^{a}$, QingLin Yang ${ }^{a}$, Fayi Song ${ }^{b}$, Nan Chen $^{a}$, Xiaoling Liao ${ }^{a}$, Bo Yao $^{a}$, \\ ShiTong Zhang ${ }^{a}$, Yanyan Chen ${ }^{c}$, and Gang Jin ${ }^{d}$ \\ ${ }^{a}$ Chongqing University of Science and Technology, Chongqing, China; ${ }^{\mathrm{b}}$ Institute of Biomaterials and \\ Biomedical Engineering, University of Toronto, Toronto, ON, Canada; 'Suzhou Institute of Nano-Tech and \\ Nano-Bionics, CAS, Suzhou, China; Institute of Mechanics, CAS, Beijing, China
}

\begin{abstract}
Carbohydrate side chain in antibody Fc region can be oxidized into aldehyde group by sodium $m$-periodate, which can simultaneously couple with the amino-group on silicon surface obtained by chemistry modification. This combination will introduce $\mathrm{C}=\mathrm{N}$ linkage and site-directed immobilization of antibody, which was confirmed by AFM observation. The acquired antibody layer can then be used for immunoassay for alpha-fetoprotein (AFP). The combined antigen is directly detected by imaging ellipsometry in grayscale format. The proposed method was further compared with commercial kit by determination 20 clinic samples with significant correlation $(R=0.93)$.
\end{abstract}

\section{ARTICLE HISTORY}

Received 7 August 2015

Accepted 3 February 2016

\section{KEYWORDS}

Alpha-fetoprotein; imaging ellipsometry; immunoassay

\section{Introduction}

Currently, immunoassay based on antigen-antibody interaction had obtained great progress in the field of nanodetection. The fact that antibody site-directed immobilization can increase antigen binding capacity has already been confirmed [1]. Although application of protein A/G for antibody immobilization is poplular [2], its blocking after immobilization is still not flawless settled because the protein A/G is non-selective to bind different antibody molecules. These shortcomings limited the protein A/G application. Now, antibody site-directed immobilization through oxidation of the sugar at the end of the Fc region into aldehyde and combination with the amino group on the chemistry modified surface became even favorable for antibody immobilization and for antigen combination [3].

A tumor marker is a substance found in the blood, urine, or body tissues that can be elevated in cancer, among other tissue types and can be used for early screening of cancer. Alpha-fetoprotein is the most effective hepatocelluar carcinoma marker [4]. Several label immunoassays including enzyme-linked immunosorbent assay (ELISA) [5], electrochemiluminescence immunoassay (ECLIA) [6] and

CONTACT Chenghong Huang chhuang2007@sinano.ac.cn.; Yanyan Chen yychen2006@sinano.ac.cn; Gang Jin Qgajin@imech.ac.cn Color versions of one or more of the figures in the article can be found online at www.tandfonline.com/ginf. 
radioimmunoassay (RIA) [7], have been applied to detect AFP. The problems for these methods using enzyme, radioactive substances, fluorescence tags or metal nano-particles, are not only dangerous to environments or operators but also loss of some biological activities of detection antibody. Therefore, label-free methods have gained some interest in the past few decades.

Biosensor based on ellipsometry(BIE), first put forward in 1995 [8], as a typical label-free technique, has high sensitivity for surface layer characterization [9] and had successively been applied to cancer marker detection [10], molecular interaction [11] and microorganism examination [12]. BIE is performed by immobilizing probe molecule onto substrate (The silicon wafers are routinely used.) to form a sensing surface, when the target of sample interacts with its corresponding probe molecule and integrates into complex on the basis of their specific interaction, which can result in molecular mass (or surface density) increase. A significant increase indicates that the sample contains target against the probe molecule whereby the existence or amount of the target in the sample can be ascertained. Furthermore, sensing areas are arranged in different places on the same substrate to form an array that can realize high throughput test [13]. In this study, oxidized anti-AFP was site-directly immoblized on the amino surface which was modifed by 3-aminopropyltriethoxysilane (APTES) as a sensing surface. The sensing surface was characterized by contact angle measurement and AFM observation. Its antigen binding activity via site-directed immobilization was also evaluated by immunoassay and simultaneously compared with physical adsorption and aldehyde crosslinking immobilization.

\section{Materials and methods}

\subsection{Materials}

Polished silicon wafers were purchased from General Research Institute for Nonferrous Metals (Beijing, China). Human AFP (from human core serum) and antihuman-AFP monoclonal antibody were obtained from Biodesign. The standard AFP was from national institution for the control of the pharmaceutical and biological products (China). Water was obtained from a millipore Milli-Q ion exchange apparatus. Aminopropyltriethoxysilane (APTES), glutaraldehyde (GA) and bovine serum albumin (BSA) were all purchased from Sigma-Aldrich. Phosphate-buffered saline (PBS, pH 7.4) was prepared in deionized water. PBST (containing $0.02 \%$ tween-20) was as flushing solution. All other chemicals were analytical grade or better. AFP test kits (ECLIA) are from Roche Company. Sera were collected from local hospital with consent for scientific use.

\subsection{Optical biosensor based on imaging ellipsometry}

The optical biosensor includes imaging ellipsometry system and array fabrication system. Imaging ellipsometry was used to visualization and quantification of the 
protein adsorption layer on the surface of silicon substrate. The light source was a Xenon lamp, and a specific collimating system was used to provide an expanded parallel probing beam with a diameter of about $25 \mathrm{~mm}$. The beam passed through a polarizer and a compensator and finally blazed on the sample surface at an incident of $70^{\circ}$. An optical filter at $632.8 \mathrm{~nm}$ wavelength was placed in the incident optical passage to select wavelength in order to increase the ellipsometric of image. The reflected beam passed through an analyzer and an imaging lens with a spatial filter located at its focus plane, and then the ellipsometry image was focused on the sensing area of a CCD camera. The reflected light is detected with a single-channel detector such as a photomultiplier or a photodiode. The working principle is: when the incident light illuminates on the different areas of the same substrate (different unit dot) resulting from the same grayscale value because the different unit spot was patterned by equal antibody solution with the same concentration, after the unit dot reacts with AFP solution, the grayscale value will increase resulting from the formation of antigen-antibody complex. The signal in grayscale format ( 8 bits, 0-255 grayscale or surface density) is measured by null ellipsometry which is based on an instrument where the polarizing elements are rotated until the signal at the detector is zero (null) and the net grayscale value can be calculated whereas the grayscale value of the control was not changed [14]. A digital image was grabbed by and stored in a computer for further evaluation by image-processing software of the program. Thereby, the content of AFP can be deduced. Detailed description for BIE detection principle is illustrated in Fig 1.

The array fabrication system was used to manufacture protein array, which was composed of sample delivery element and protein array reactor element. The sample delivery element includes a multiple micro-fluidic channel dispensing pump that can convey sample solutions by multiple parallel channels using the Teflon tubes connection under the conditions that the negative pressure was generated. Antibody solution was patterned on the substrate to form different unit dot in array format, each dot may function as capture layer. The reactor in details can be reffered

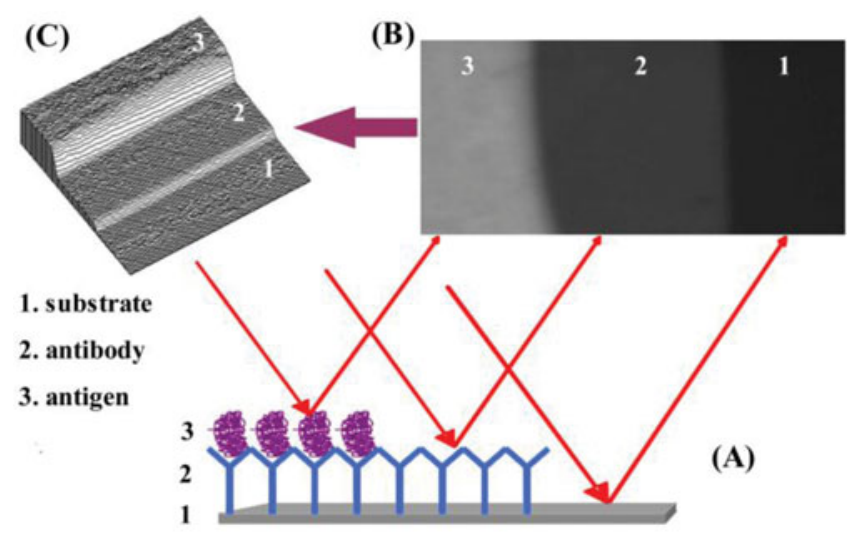

Figure 1. Detection principle illustration of optical biosensor based on imaging ellipsometry(A), obtained grayscale image (B) and corresponding three dimension map (C). 
to literature [15]. In this way, the optical biosensor can effectuate high through-put detection by multiple protein dots on the same substrate with a single experiment.

\subsection{Surface modification}

The Polished silicon wafers were cut into rectangles about $1.5 \times 2 \mathrm{~cm}$ before used. The prepared silicon wafers were then immersed into the solution $(30 \%$ $\mathrm{H}_{2} \mathrm{O}_{2}: 98 \% \mathrm{H}_{2} \mathrm{SO}_{4}=1: 3 \mathrm{v} / \mathrm{v}$ ) to oxidize 30 minutes. After rinsed by deionized water and ethanol, the washed silicon wafers were incubated in a mixture APTES and ethanol $(1: 4, \mathrm{v} / \mathrm{v})$ for 2 hours. The above reaction is the $\mathrm{NH}_{2}$ group immobilization process, forming a layer of densely packed $\mathrm{NH}_{2}$ groups on silicon dioxide layer. Later, the silicon wafers were rinsed by ethanol 3 times and phosphate buffered saline (PBS) 3 times. The silicon wafers were at last placed into a mixture of glutaraldehyde and PBS (1:10, v/v), shaken lightly in a shaker for $1 \mathrm{~h}$, and finally, washed by PBS buffer 3 times. The amino group carrying lysine of the antibody molecules will covalently combined with the substrate surface. The silicon wafer was left in a beaker with PBS buffer until use. The surface modification was characterized by contact angle measurements (Sessile drop method).

\subsection{Antibody oxidization and site-directed immobilization}

In brief, a $3 \mathrm{mg}$ aliquot of the rat anti-AFP monoclonal antibody was dissolved in $1 \mathrm{ml} 0.5 \mathrm{M}$ sodium acetate buffer, $\mathrm{pH} 5.2$, to which was added $1 \mathrm{ml} 20 \mathrm{mM} \mathrm{NaIO}_{4}$ solution. The reaction was allowed to take place for $1 \mathrm{~h}$ at room temperature with shaking. Excess $\mathrm{NaIO}_{4}$ was then separated from the reaction mixture by dialysis at $4^{\circ} \mathrm{C}$ for $2 \mathrm{~h}$ with 2.0 -liter portion $\mathrm{pH} 5.2,20 \mathrm{mM}$ acetate buffer containing $0.15 \mathrm{M}$ sodium chloride [16]. It was followed by three additionaldialysis, (and) each performed for $2 \mathrm{~h}$. The oxidized antibody was directly deposited onto modified silicon wafer to react 20 minutes. After coupling reaction, the surface was blocked by BSA for 30 minutes. Later, the surface was rinsed by flushing solution and delivered to AFP detection. AFM observation was performed using a commercial system (Nanoscope IIIa, Digital Instruments, Santa Barbara) in contact mode. A 16um scanner was used for surface inspection. Soft cantilevers were $200 \mathrm{um}$ long with an integrated pyramidal Si3N4 tip with a spring constant of $0.12 \mathrm{~N} / \mathrm{m}$. Typical forces for all measurements were of the order of approximately $1 \mathrm{nN}$ or less.

In order to get insight into site-directed immobilization, we concurrently conducted other antibody immobilization methods including antibody direct adsorption onto the $-\mathrm{NH}_{2}$ surface and antibody covalent immobilization through glutaraldehyde combination for the next immunoassay.

\subsection{Immunoassay}

Solutions (serum sample or calibrator) containing different concentration AFP flow independently through the sensing surface in array to react 20 minutes (Flow rate: 
$1.0 \mu \mathrm{L} / \mathrm{min}$ ). This process permits the immobilized antibodies of sensing surface to catch the target antigen (here is AFP). Then, PBS was used to flush the unbound antigen away for 5 minutes (Flow rate: $10.0 \mu \mathrm{l} / \mathrm{min}$ ). Finally, all of the protein dots were flushed by PBS and pure water several times in sequence and send to imaging ellipsometry examination after drying under a stream of nitrogen. The blank control without antigen was set at the same time.

\subsection{AFP analysis by commercial kit}

The procedure about commercial kit measurement was strictly complied with the specification offered by manufacturer. The whole process was completed at room temperature in a professional laboratory. Total 10 patients and 10 healthy sera were analyzed. Each sample was determined two times and the average value was calculated.

\section{Results and discussions}

\subsection{Silicon modification}

The cleaned silicon wafers (contact angle $<5^{\circ}$ ) oxidized by $\mathrm{H}_{2} \mathrm{SO}_{4} / \mathrm{H}_{2} \mathrm{O}_{2}$ solution will react with APTES yielding amino group with positive charges (contact angle 44 $\pm 1.6^{\circ}$ ) and the latter will stepwise link with the GA to produce a surface (contact angle $55 \pm 2^{\circ}$ ) carrying free adlehyde groups. The high reactive aldehyde surface can form imine linkages with the primary amine group of lysine residue which randomly present in the antibody molecular.

\subsection{Antibody immobilization and AFM observation}

AFM has been extensively used to image biological system such as bacterial [17], virus [18], and interaction of antigen-antibody [19]. We scanned the silicon wafer surface $\left(-\mathrm{NH}_{2} /-\mathrm{CHO}\right.$ surface) and revealed a highly flat and smooth surface with an average roughness less than $0.5 \mathrm{~nm}$, indicating that the surface will present features if the antibody was patterned on it. Figure 2 shows AFM images obtained different chemical modification. It can be seen from the image that the antibody molecules were adsorbed in the form of clusters instead of disperse location. The height of these clusters was 3.0-3.3nm varied from 20.0 to $50.0 \mathrm{~nm}$ in lateral size, indicating the formation of nonuniform surface aggregates. The antibodis will aggregate into cluster onto the hydrophilic surface when blew by a stream of N2 observed by AFM [20] because of the weak interaction forces. On the contrary, the strong combination on hydrophobic surface made the interaction firm and the antibodies will not aggregate into clusters without observed by AFM [21]. Here, the antibody spread homogeneously on the silicon surface confirmed by AFM observation. This comfired strong interaction between antibody molecular and the $-\mathrm{NH}_{2}$ surface. The carbohydrate of the Fc region can be oxidized into aldehyde group to 

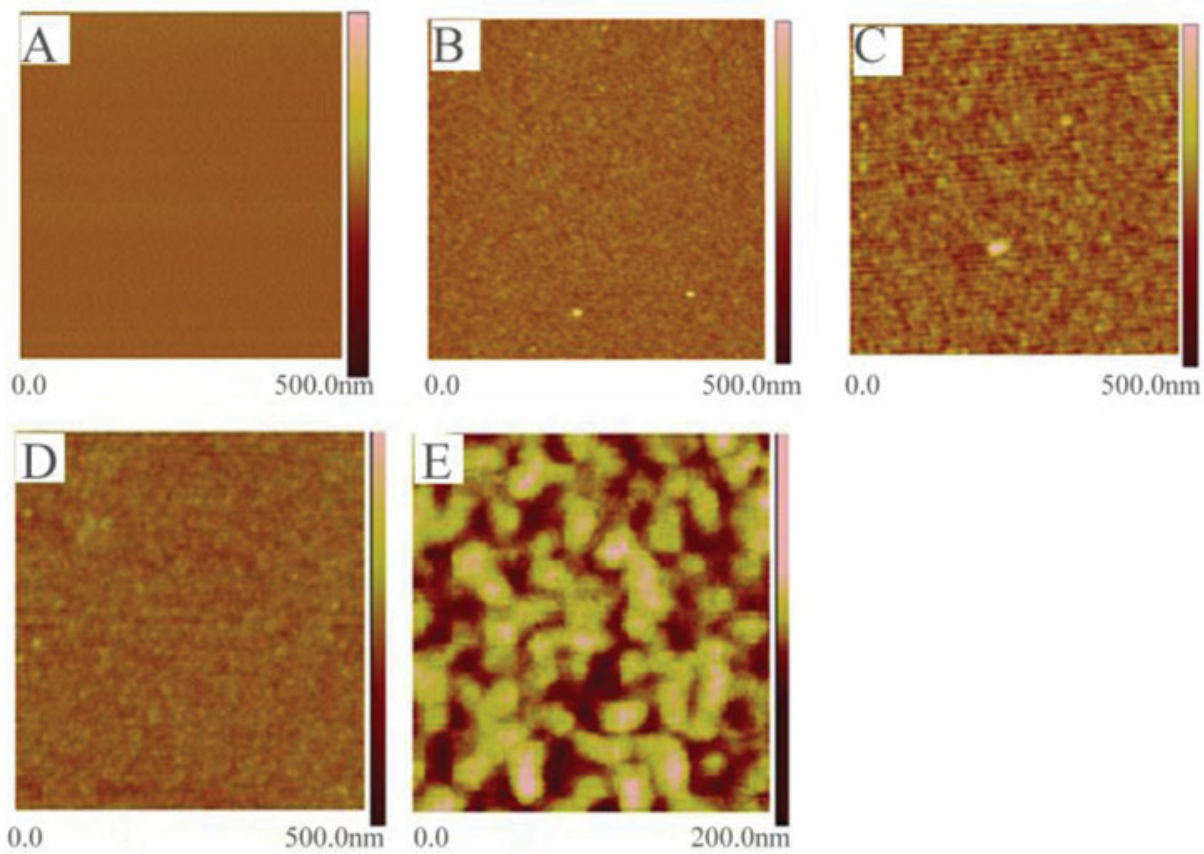

Figure 2. AFM images for chemical modification and site-directed antibody immobilization. (A)-OH surface, $-\mathrm{NH} 2$ surface, $-\mathrm{CHO}$ surface, immobilized antibody on $-\mathrm{CHO}$ surface $(\mathrm{C})$ and enlarged image (D).

form imine linkage with lysine group in antibody. This linkage fastens antibody stably on the surface and the Fab fragment of the antibody molecular will direct outside of the surface. This kind of fixation favors antigen binding.

\subsection{AFP immunoassay}

Standard serum sample containing AFP was diluted to different concentration from $10.0 \mu \mathrm{g} / \mathrm{ml}$ to $1.0 \mathrm{ng} / \mathrm{ml}$, and then reacted with anti-AFP for 30mintues. With formation of anti-AFP and antigen complex, the thickness of protein layer on the silicon surface changes can be expressed as grayscale value changes. The different protein dots on the array with different grayscale value could be automatically captured and processed in digital images (Fig 3). As expected, the grayscale value with higher concentration is more obvious than with lower concentration and the protein dot appears more lightness accordingly. The grayscale value plotted to logarithm of AFP concentration can be regressed and represented by the equation of $\mathrm{y}=3.29 \log \left(\mathrm{C}_{\mathrm{AFP}}\right)+91.8\left(\mathrm{R}^{2}=0.98\right.$, Fig 4$)$. Therefore, AFP can be quantitatively detected by the optical biosensor. The heaptocellular carcinoma has a high occurrence in the east of Asia, especially in China. The AFP content of the healthy adult serum is often not more than $25.0 \mathrm{ng} / \mathrm{ml}$. We concern the signal increase at this cut-off value. The net grayscale value is 15.2 compared to the blank control and can completely be applied to positive decision. 


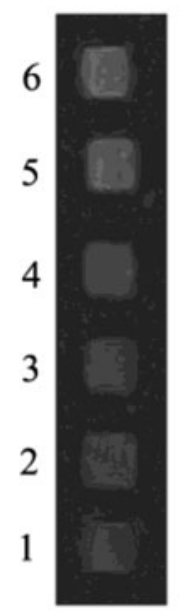

(A)

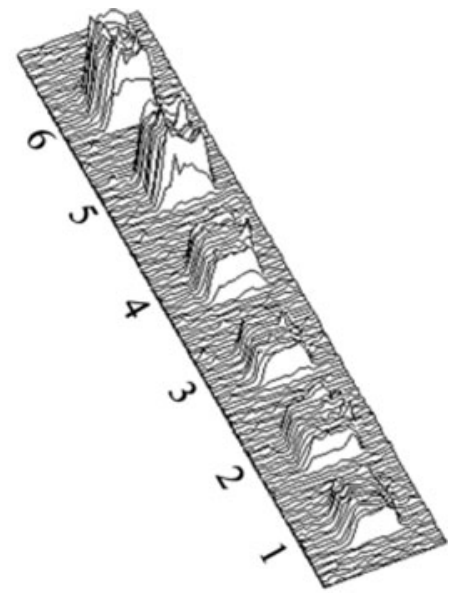

(B)

Figure 3. Typical ellipsometry images of the protein dots obtained by serial diluted AFP (A) and corresponding three dimension map (B). Dots from 2-6 were 10-diluted AFP and dot 1 was blank control.

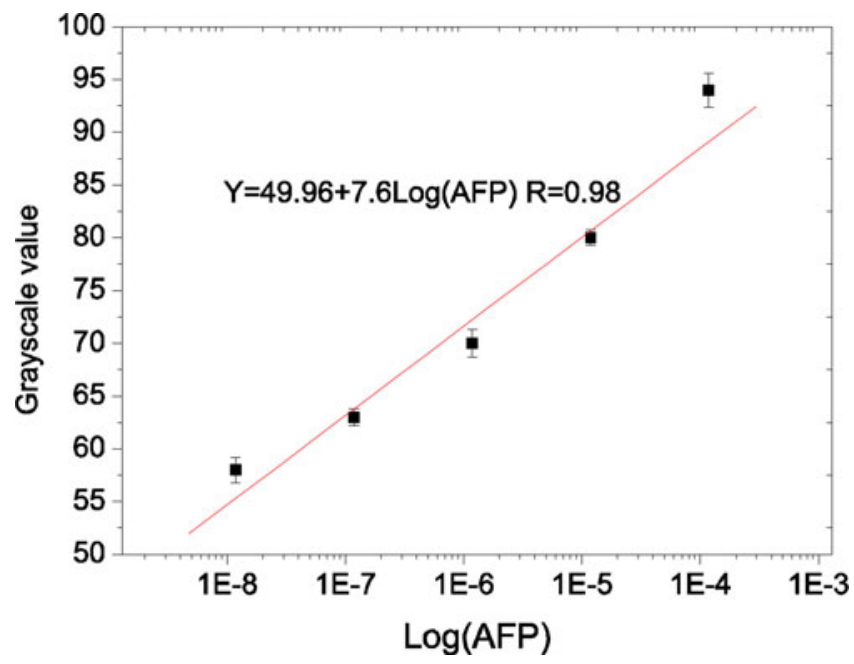

Figure 4. Typical dose-response curve obtained by serial diluted AFP.

\subsection{Methodology comparison}

The surface densities for three antibody immobilization methods and antigen combination were listed in table 1 . As can be seen, the antibody immobilized amount

Table 1. Grayscale values for antibody immobilization and antigen binding with different chemical surfaces.

\begin{tabular}{lccc}
\hline & $-\mathrm{NH}_{2}$ surface & -CHO surface & Site-directed immobilization \\
\hline Antibody immobilization & $46.8 \pm 2.6$ & $47.7 \pm 2.1$ & $44.8 \pm 2.4$ \\
Antigen combination & $52.4 \pm 1.8$ & $56.8 \pm 2.8$ & $60.8 \pm 1.9$ \\
\hline
\end{tabular}




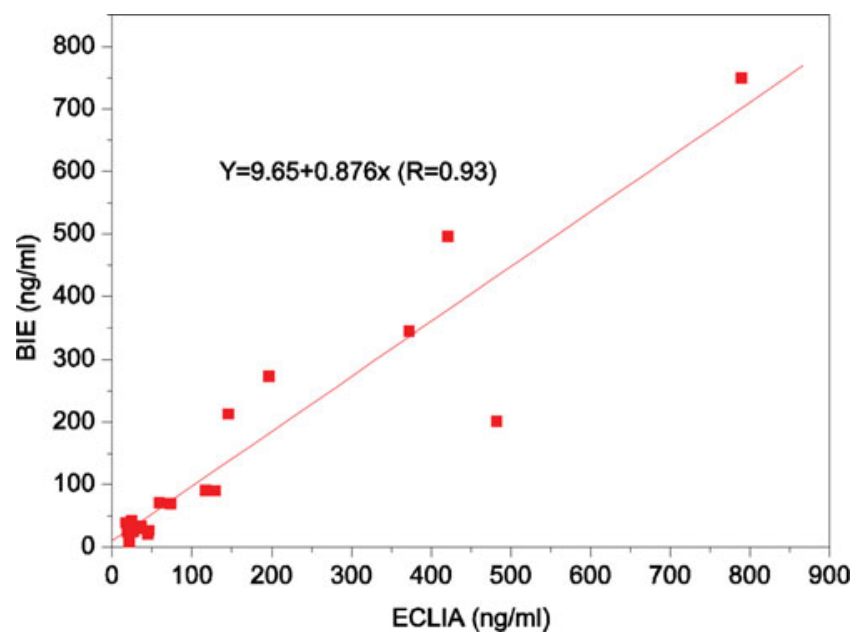

Figure 5. Correlation between ECLIA and BIE method was analyzed by 20 clinic samples.

$(100 \mu \mathrm{g} / \mathrm{ml}$ anti-AFP was used.) and antigen combining amount $(1.0 \mu \mathrm{g} / \mathrm{ml} \mathrm{AFP} \mathrm{was}$ used.) for site-directed immobilization were enhanced by $32 \%$ and $16 \%$ compared with physical adsorption (- $\mathrm{NH}_{2}$ surface) and aldehyde combination (-CHO surface). The latter two can be contributed to antibody random arrangement and steric hindrance to influence its antigen binding capacity. We further tested 10 healthy and 10 patient sera which had already been validated by pathology examination. It thoroughly coincides with the results of ECLIA $(\mathrm{R}=0.93)$ by correlation analysis.

\section{Conclusion}

The carbohydrate of anti-AFP can be oxidized into aldehyde and covalently linked with the APTES modified amino surface, which was confirmed by AFM observation. This favors antibody Fab outside to the surface and increases the antigen binding capacity. Compared with amino and aldehyde surface, the capacities of antibody immobilization amount and antigen binding of site-directed immobilization were enhanced by $32 \%$ and $16 \%$, respectively. The dose-response curve showed that AFP can be quantitatively detected after logarithm transformation. It demonstrated that the optical biosensor can be used in hepatocellular carcinoma screening.

\section{Acknowledgments}

This work is supported by the cooperative project of academician workstation of Chongqing University of Science \& Technology (CKYS2014Y5), Research Foundation of Chongqing University of Science \& Technology (CK2014B4) and Chongqing pogram of Application Foundation and Advanced Technology(cstc2014jcyjA50032, cstc2014jcyjA10029).

\section{References}

1. P. Batalla, C. Mateo, and V. Grazu et al., Immobilization of antibodies through the surface regions having the highest density in lysine groups on finally inert support surfaces. Process Biochem. 44, 365-368(2009). 
2. G. Demirel, M. O. Çağlayan, and B. Garipcanet al., Oriented immobilization of IgG on hydroxylated $\mathrm{Si}(001)$ surfaces via protein-A by a multiple-step process based on a selfassembly approach Journal of Materials Science. 42, 21-25 (2007).

3. H. K. Jung, Y. H. Sang, and S. H. Han et al., Improving immunobinding using oriented immobilization of an oxidized antibody. Journal of Chromatography A.1161, 9-14 (2007).

4. M. Lorinda, J. T. Wright, and C. J. F Kreikemeier. A concise review of serum markers for hepatocellular cancer. Cancer Detection and Prevention. 31, 35-44 (2007).

5. W. M, B. S. Kwan. An enzyme-labelled immunoassay for the measurement of alphafetoprotein La Ricerca Clin Lab; 12, 301-306 (1982).

6. S. Yuan, R. Yuan, and Y. Chai, et al. Sandwich-type electrochemiluminescence immunosensor based on Ru-silica@Au composite nanoparticles labeled anti-AFP Talanta.82, 1468-1471 (2010).

7. P. J. Johnson, B. Portmann, and R. Williams, Alpha-fetoprotein concentrations measured by radioimmunoassay in diagnosing and excluding hepatocellular carcinoma. British medical Journal. 661-663 (1978).

8. G. Jin, P. Tengvall, and I. Lundström et al., A Biosensor Concept Based on Imaging Ellipsometry for Visualization of Biomolecular Interactions. Ana Biochem; 232, 69-72 (1995).

9. G. Jin, J. R., H. A. Imaging ellipsometry revisiteda developments for visualization of thin transparent layers on silicon substrates. Rev Sci Instrum. 67, 2930-2936 (1996).

10. C. H. Huang, Y. Y. Chen, and Z. H. Wang ET AL., Detection of alpha-fetoprotein through biological signal amplification by biosensor based on imaging ellipsometry. Thin Solid Films; 519, 2763-2767 (2011).

11. J.Zhu, X. Y. Duan, and X. M.Guo et al.,A human SARS-CoV neutralizing antibody against epitope on S2 protein. Biochem and Biophy Res Comm. 333, 186-193 (2005).

12. C. H.Huang, J. X. Li, and Y. Tang., Detection of duck hepatitis virus serotype1 by biosensor based on imaging ellipsometry. Current Applied Physics.11, 353-357 (2011).

13. Z. H. Wang, Y. H. Meng, and P. Q. Ying et al., A label-free protein microfluidic array for parallel immunoassays. Electrophoresis. 27, 4078-4085 (2006).

14. Y. Y. Chen, Y. H. Meng, and G. Jin. Optimization of off-null ellipsometry for air/solid interfaces Applied Optics; 46, 8475-8481 (2007).

15. Z. H.Wang, Y. H.Meng, and P. Q.Ying et al., A label-free protein microfluidic array for parallel immunoassays. Electrophoresis; 27, 4078-4085 (2006).

16. Q. W. Ping, W. Lei, and W. C.Xiao et al. Orientation of Antibodies on a 3-APTES-Modified Silicon Wafer Surface. Journal of Inclusion Phenomena and Macrocyclic Chemistry. 35, 4194291 (999).

17. H. S. Satoshi, T. M. Takao, and, M. Abe et al.,A new method of biosensing with $1 \mu \mathrm{l}$ of Escherichia coli suspension using atomic force microscopy. Ana Biochem; 345, 116-121 (2005).

18. E. V. Dubrovin, Y. F. Drygin, and DS, V. K.Novikov et al., Atomic force microscopy as a tool of inspection of viral infection. Nanomedicine: Nanotechnology, Biology, and Medicine. 3, 128131 (2007).

19. J. Wakayam, S. Akanuma, and T. Ohtani et al., Methods for reducing nonspecific interaction in antibody-antigen assay via atomic force micros. Ana Biochem. 380, 51-58 (2008).

20. X. Z. Xu, C. Grant, and R. Jian et al., Orientation of a Monoclonal Antibody Adsorbed at the Solid/Solution Interface: A Combined Study Using Atomic Force Microscopy and Neutron Reflectivity. Langmuir; 22, 6313-6320(2006).

21. F. Rusmini, Z. Zhong, and J. Feijen. Protein Immobilization Strategies for Protein Biochips. Biomacromolecules. 8, 1775-1789 (2007). 